\title{
Rain Gutter Detection in Aerial Images for Aedes aegypti Mosquito Prevention
}

\author{
Lucas Rossi ${ }^{1}$, André R. Backes ${ }^{1}$, Jefferson R. Souza ${ }^{1}$ \\ ${ }^{1}$ School of Computer Science, Federal University of Uberlândia, Brazil \\ arbackes@yahoo.com.br
}

\begin{abstract}
The detection of Aedes aegypti mosquito is essential in the prevention process of serious diseases such as dengue, yellow fever, chikungunya, and Zika virus. Common approaches consist of surveillance agents who need to enter residences to find and eliminate these outbreaks, but often they are unable to do this work due to the absence or resistance of the resident. This paper proposes an automatic system that uses aerial images obtained through a camera coupled from an Unmanned Aerial Vehicle (UAV) to identify rain gutters from a shed that may be mosquitoes' foci. We use Digital Image Processing (DIP) techniques to differentiate the objects that may or may not be those foci of the mosquito-breeding. The experimental results show that the system is capable of automatically detecting the appropriately mosquito-breeding location.
\end{abstract}

Keywords-Aedes aegypti, aerial images, image processing.

\section{INTRODUCTION}

In Brazil, in 2016, more than one million cases of the three central diseases transmitted by the Aedes aegypti mosquito (Dengue, Zika, and Chikungunya) were recorded. The number of reported deaths reached 794, among them, 629 per dengue, 159 per chikungunya and six per Zika [1]. In 2018 were 32,161 probable cases of dengue in the country, 132 cases of Dengue with signs of alarm and a confirmed death in the State of Paraíba. The regions with the highest incidence of probable cases are the regions: Southeast (40.2\% of occurrences) and Center-West $(32.5 \%)$. During this same period, 705 probable cases of Zika virus fever, 7,406 of Chikungunya fever were registered, one confirmed death and seven other deaths under investigation [2].

In Uberlândia, in the year 2016, up to the epidemiological week 50, 12,949 dengue cases were reported. In the year 2017, there was a decrease of $82 \%$ concerning the previous year, with 3,747 cases reported [3]. In 2018, 335 cases of dengue, five of Chikungunya and four of Zika were reported by epidemiological week [4].

The life cycle of Aedes Aegypti begins after the laying of eggs by a female on the wall of a breeding ground with water (eggs are not deposited directly in water). Such eggs can remain without hatching for a long time, are resistant to dryness and can last up to 450 days. After the egg hatch, the larval stage of the mosquito begins, the larva feeds mainly on the organic matter present in the breeding ground. After about five days the pupal phase begins, this period lasts on average three days, during which time the pupa remains on the surface of the water to facilitate the flight as an adult. It is during the adult phase that mosquito can transmit diseases to man [5].
According to a survey carried out by the Ministry of Health in 2013, $90 \%$ of mosquitoes are found in homes, and in 45 days a mosquito can contaminate up to 300 people [6]. Therefore, there are monitoring agents able for the houses to prevent the reproduction of the insect by removing and destroying objects that could become mosquito breeding sites. Some of the objects that can be Aedes aegypti breeding are: plant pots, dumpsters, plants that accumulate water, bottle caps, eggshell, cans, plastic bags, glass containers, disposable cups, lakes, waterfalls, water tanks, abandoned old tires, PET and glass bottles, shards of glass on the walls, buckets [7].

The contribution of this work is the detection of objects to be breeding sites of the Aedes Aegypti mosquito, which may be difficult to access for inspection agents or even for the population (rain gutters, Figure 1). The objective is to use the images captured by the camera coupled to the UAV to identify if the object or location is a possible focus of the mosquito, to carry out the preventive actions in the place and to reduce the chances of transmission of the diseases transmitted by it.

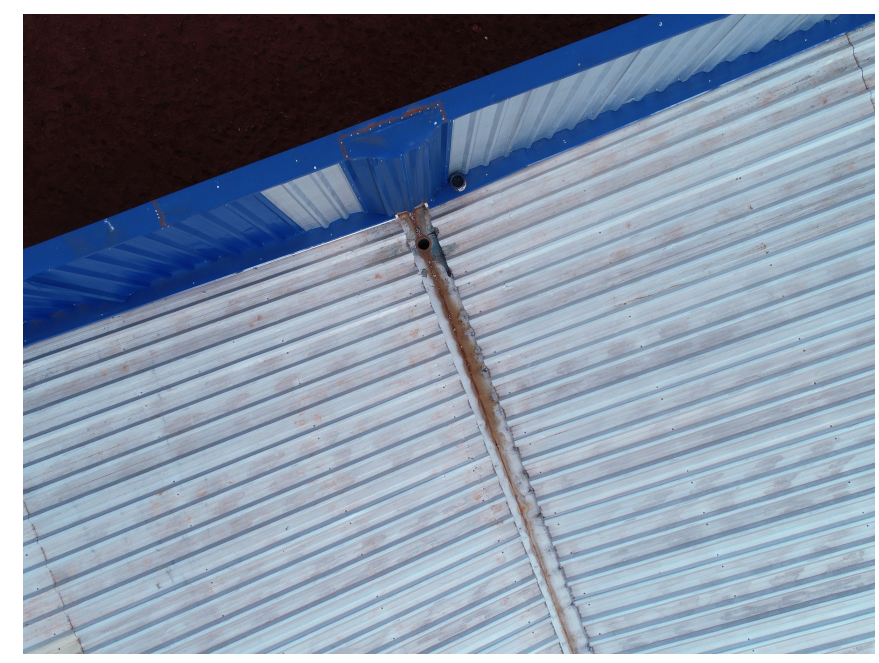

Fig. 1. Example of an image with rain gutters captured with a UAV.

The remainder of this paper is organized as follows: Section II discusses the related work to the Aedes aegypti mosquito detection; material and methods is shown in Section III] the proposed methodology is presented in Section IV the results and analysis are shown in Section V; finally, Section VI concludes and suggests directions for future work. 


\section{RELATED WORK}

In [8], the authors offer a method for the identification of mosquito breeding sites using wireless networking and the removal of stagnant water through electromechanical pumping systems. The inactive water areas are identified and reported by users using a web-based portal. A vehicle was carrying a Global Positioning System (GPS), on-board Camera and a pumping system with a tank for removing the stagnant water. Finally, they removed stagnant water using a pumping system. The results show the effectiveness of the proposed approach.

In [9], it is shown an approach for detecting the presence of stagnant water bodies in images obtained in settings. Stagnant water can become sites for mosquitos to grow, such as Aedes aegypti. They present a method that can identify puddles in these images with about $88 \%$ successfully accuracy. The method is robust to image focus, making it a good choice for datasets produced by different kinds of cameras, including UAVs.

The work proposed by [10] uses crowdsensing techniques coupled with the medical professional's diagnosis of Zika to impute data to provide a location for Zika outbreaks. Results show that the approach has the potential to create impactful results. If adequately tested this system helps prevent Zika infections.

Dengue is one of the rapidly spreading and deadly diseases in Sri Lanka. A UAV was used to capture the mosquito breeding. It can inspect both accessible and inaccessible places to a human. [11] shows an approach to detect dengue mosquito via UAV images. The method captures the images of the water retention areas. Results produced on the field test were satisfactory of accuracy in identifying water retention areas.

Annually thousands of people die from dengue fever, chikungunya, and Zika. The majority of mosquito-breeding are bottles, tires, barrels, or any stagnant water. The work proposed by [12] shows a system to aid the mosquito-breeding habitats employing computer vision on aerial images. Initially, a dataset was created with video sequences from a UAV and the manual annotation in several scenarios. The features extracted from the images were HSV color space, histograms, and edge detection to train a random forest classifier. Results demonstrate that the classifier resulted in an accuracy higher than $99 \%$ in the test set. Then, the system is capable of automatically determining the GPS coordinates of the mosquito breeding location.

\section{MAterial AND Methods}

\section{A. Image acquisition}

For image acquisition, we used DroneDeploy software to upload a map with a predefined route that must be covered by the UAV. The UAV flies over the route and takes pictures, which are uploaded to a computer after the flight and merged into an image containing the whole area observed. To obtain the aerial images, we used an aircraft Phantom 4 Pro. Aerial images were captured by an onboard camera of $4864 \times 3648$ pixel resolution and $72 \mathrm{dpi}$, flying at an altitude of 30 meters. The dataset consists of 207 images captured by the UAV.

\section{B. Mathematical morphology}

Proposed by Jean Serra in its Ph.D. thesis, mathematical morphology describes different techniques to process digital images using many concepts from set theory [13]. Mathematical morphology is usually applied to binary images, where each image is defined as a subset of a two-dimensional integer grid, $\mathbb{Z}^{2}$. Given an input image, we desire to process; mathematical morphology requires a second binary image of pre-defined shape called a structuring element. It is the structuring element that guides how the input image will be processed, analyzed and/or have extracted its geometrical structures. In the following paragraphs we describe basic mathematical morphology operations used in this work:

Dilation: Given an input binary image $A$ and a structuring element $B$, this operation increases the area of the objects in $A$ according to the shape of an element $B$, as shown in Equation 1 . Depending of the shape and size of $B$, different objects in $A$ may be fused into a single one.

$$
A \oplus B=\left\{x \in Z^{2} \mid c=a+b, a \in A \wedge b \in C\right\}
$$

Connected component labeling: this process groups image pixels based on their connectivity and similar intensity. Basically, for a binary image, this method scans the image and attributes the same label to all foreground pixels that are in some way connected with each other. Each label represents a different and separated structure in the original image.

\section{HSV color space}

In general, acquisition devices capture images in the RGB color space due to its simplicity and its ability to satisfactorily expresses the captured scene. Nevertheless, RGB space does not reproduce how humans interpret color, in particular, the luminance and the chrominance of the color. Depending on the application, HSV color space emerges as an alternative to represent color. Three components define HSV model: hue $(\mathrm{H})$, the color component; saturation (S), the amount of gray; and value $(\mathrm{V})$, the brightness or intensity of the color. This is a perceptual color model which mimics human color response, i.e., it models the color in a way that is closer to how human vision perceives color attributes [14].

\section{Hough Transform}

Developed by Paul Hough in 1962, Hough transform is an essential tool to detect parametric objects in digital images such as lines, circles, and ellipses. It is usually applied in a preprocessed image, for example, in the resulting image of a border detection method.

Basically, the Hough transform maps an image pixel into a parametric space organized as an $n$-dimensional accumulator. For the case of line detection, each pixel $(x, y)$ of the object has a parametric line $y=a * x+b$ associated to it, where $a$ is the slope, and $b$ is the intercept.

Duda e Hart [15] showed that it is possible to fully represent these lines using polar coordinates, where each line is defined by its length, $r$, and orientation, $\theta$, of the normal vector to the original line: 


$$
r=x * \cos (\theta)+y * \sin (\theta)
$$

By using this Equation 2 each point of the original image is mapped into a sine curve in the polar space. Different aspects of the object in the original image result in different sine curves that intercept into a single point at the polar space, and each $(r, \theta)$ point in polar space represents a line in the original image. These properties enable us to detect the line equation of the original space by identifying the point where most sine curves intercept each other in the polar space.

\section{Proposed Methodology}

In this section, we present the proposed methodology to detect rain gutter on shed roofs. Usually, it is possible to detect the rain gutter by detecting other materials accumulated in its structure, such as soil residue, leaves etc. To accomplish this task, we first convert the image from RGB to HSV color space. Then, applied a threshold over hue $(H)$ and saturation $(S)$ channels to extract objects that may indicate the presence of a rain gutter in the image. Due to the characteristics of the region, we used the following equation to convert the hue and saturation of the input image into a binary image $B$ :

$$
B_{i, j}= \begin{cases}1 & \text { if }\left(H_{i, j} \geq 0.86 \vee H_{i, j} \leq 0.20\right) \wedge S_{i, j} \geq 0.20 \\ 0 & \text { otherwise }\end{cases}
$$

where, $H, S \in[0,1]$ and $i, j$ represent a pixel in the image. These values were manually defined to select the more reddish shades in the image. In the sequence, we performed a dilation of the binary image $B$ using a disk of radius $r=10$ as structuring element. The dilation is necessary as the HSV segmentation may result in a disconnected rain gutter and, as we don't know the orientation of the gutter previously, the disk is the better option to connect nearby elements in all directions. Figure 2 (a)-(c) shows the result of the segmentation and the dilation process.

The segmentation process may result in multiples elements. Since the rain gutter is a thin and elongated structure, characteristics such its area and its aspect ratio are useful to discriminate it from other objects. We used connected component labeling to identify each object in the dilated image. The elements are sorted according to its area value, i.e., the number of pixels contained in that object. Empirical analysis showed that the best candidates to rain gutter are objects whose area is smaller than $3 \%$ of the image. Starting from the largest to the smallest component, we selected the largest component whose area falls into this criteria. Figure 2(d) shows an example of the largest connected component in an image.

In the next step, we verify if the selected object is a rain gutter. To accomplish that we computed the bounding rectangle of the selected object and used the rectangle sides to calculate the object's aspect ratio and the rectangle area, as shown in Figure 2(e). We defined the aspect ratio as

$$
a r=100 * A / B
$$

where $A$ and $B$ are, respectively, the smallest and largest sides of the rectangle. We consider any object as a rain gutter if the aspect ratio is equal to or greater than $21 \%$ and its area equals to or smaller than $10 \%$ of the area of the bounding rectangle. If the object fails into fitting these criteria, it is discarded, and the next object is evaluated. This process ends when we find an object fitting both criteria our when there are no other objects to test. Finally, we use the Hough transform to compute the line segment which corresponds to the object selected and use this information to highlight the rain gutter in the image, as shown in Figure 2 (f).

\section{EXPERIMENTAL RESULTS}

We applied the proposed methodology in a set of 207 images captured by the UAV. From these, only 56 images presented a rain gutter. Remaining images consisted of images of shed roofs without the presence of rain gutter or images of the region near the building, which consists basically of terrain. Figure 3 shows examples of the three situations.

Using our approach, we were able to detect rain gutters in 60 images from which 51 images had rain gutters that were effectively identified while 9 are the false positive results. In 5 images, our approach identified was not able to detect the existing rain gutters or it identified erroneous other structure. It is crucial to emphasize that some missed rain gutters are in images that contain only a small portion of the shed roof, so that the structure we aim to detect is extremely small, hinders the detection process. Some cases of the false positive are due to presence of larger structures that fit into the parameters defined to detect rain gutters.

One example of this situation is shown in Figure 4. This figure shows the presence of a wire-like structure in the roof shed which is falsely detected as a rain gutter. As one can see, this structure presents a length and aspect ratio similar to our target objects, which explains its detection. This situation indicates that a post-processing step may still be necessary to filter false candidates.

Figure 5 shows some examples of the correct detections. Figure 5(a) is the easiest case as the target structure is well isolated and it is the largest connected component detected by our approach. Figure 5(b) displays a large amount of terrain in the image. Initially, our approach considers this terrain as our target structure. However, since this object doesn't fit the established parameters of our algorithm, it is discarded, and other structures are evaluated until one fits (the small structure at the top right corner). Finally, Figure 5.c) shows a situation where both rain gutter and terrain are feasible candidates after the segmentation. In this particular case, the object which corresponds to terrain is discarded when we evaluate its bounding rectangle and notice its aspect ratio fails to our given criteria.

\section{CONCLUSIONS}

We propose a simple detection of objects to be breeding sites of the Aedes Aegypti mosquito aiming to prevent several types of diseases, such as dengue fever, yellow fever, chikungunya, 


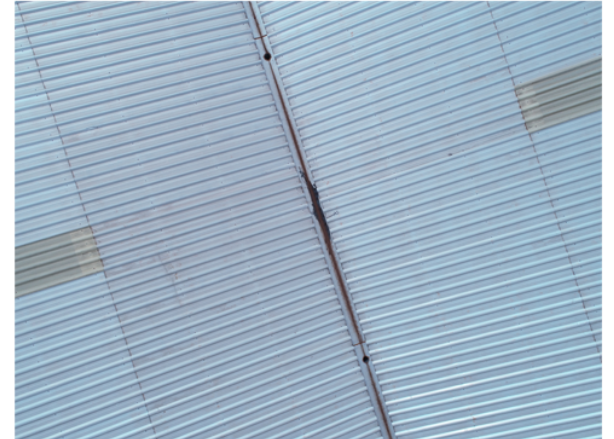

(a)

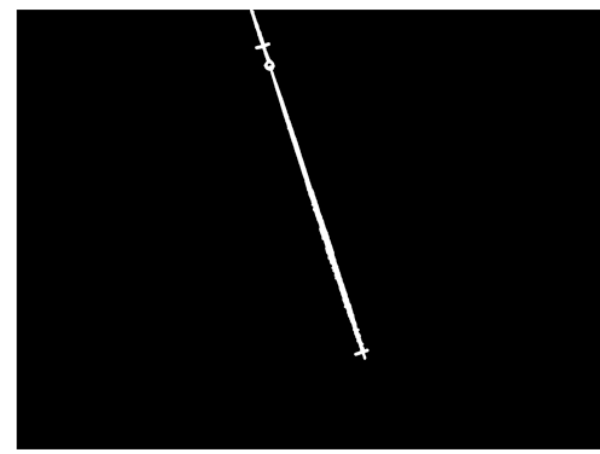

(d)

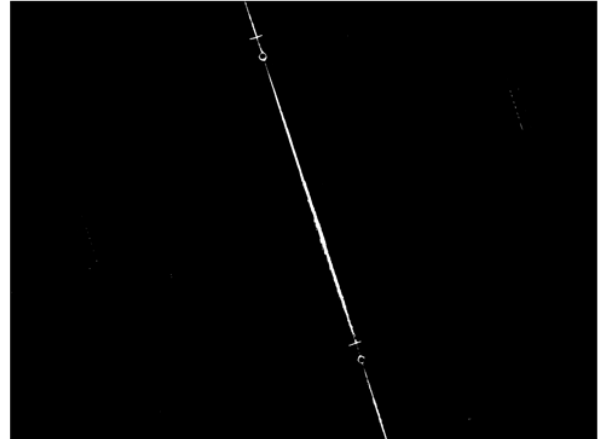

(b)

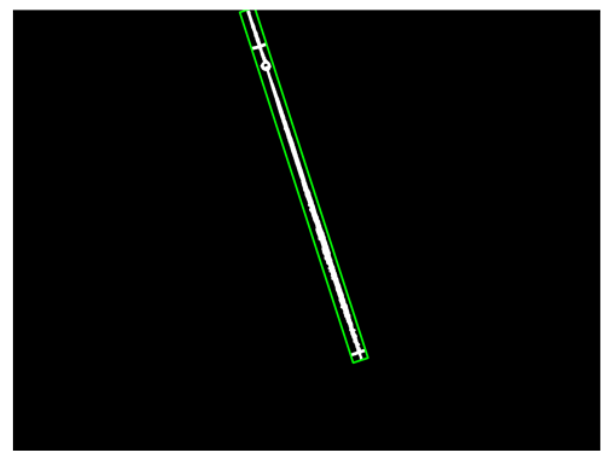

(e)

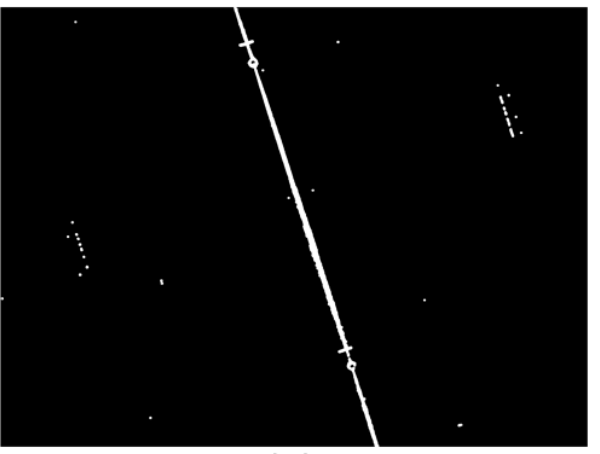

(c)

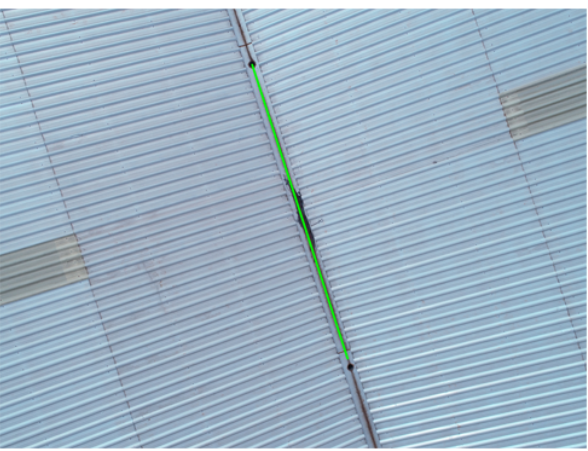

(f)

Fig. 2. Rain gutter candidate detection process: (a) Original image; (b) HSV segmentation; (c) Dilation of the segmented image; (d) Largest connected component; (e) Bounding rectangle; (f) Line detected using Hough transform.

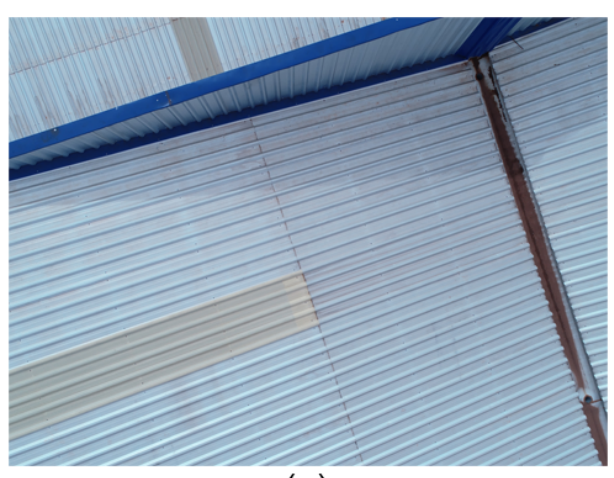

(a)

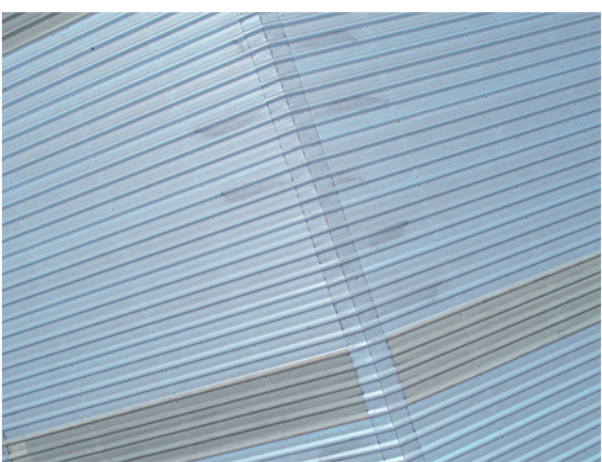

(b)

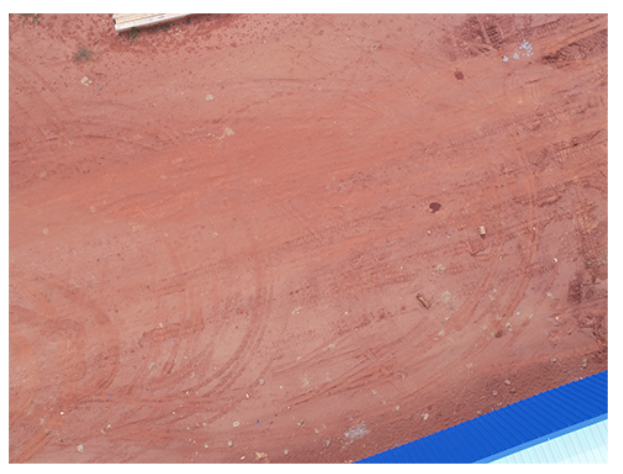

(c)

Fig. 3. Examples of the dataset: (a) shed roofs with rain gutter; (b) shed roofs without rain gutter; (c) terrain near building

and Zika virus. The purpose is to use the images captured by the camera coupled to the UAV to identify if the location is a possible focus of the Aedes Aegypti mosquito, to carry out the preventive actions in the place and to reduce the chances of transmission of the diseases transmitted by it.

We believe the contributions made in this paper are an essential step towards the prevention of the Aedes Aegypti mosquito. Also, results demonstrate that the system is capable of automatically detecting the appropriately mosquito-breeding location. Lastly, false positive results can be handled on postprocessing step useful to filter false candidates.

As future work, we will consider more objects to be explored aiming the prevention of the Aedes Aegypti mosquito. Objects as water tanks, swimming pool, abandoned old tires, and glass bottles. Furthermore, we also intend to apply machine learning techniques to compare with the proposed approach of this work.

\section{ACKNOWLEDGMENT}

The authors would like to acknowledge the Federal University of Uberlândia, FAPEMIG (Minas Gerais Research Foundation) under Grant \#APQ-03437-15, CNPq (National Council for Scientific and Technological Development) under Grant \#400699/2016-8, \#302416/2015-3 and \#301715/2018-1, and PROPP-UFU. This study was financed in part by the 


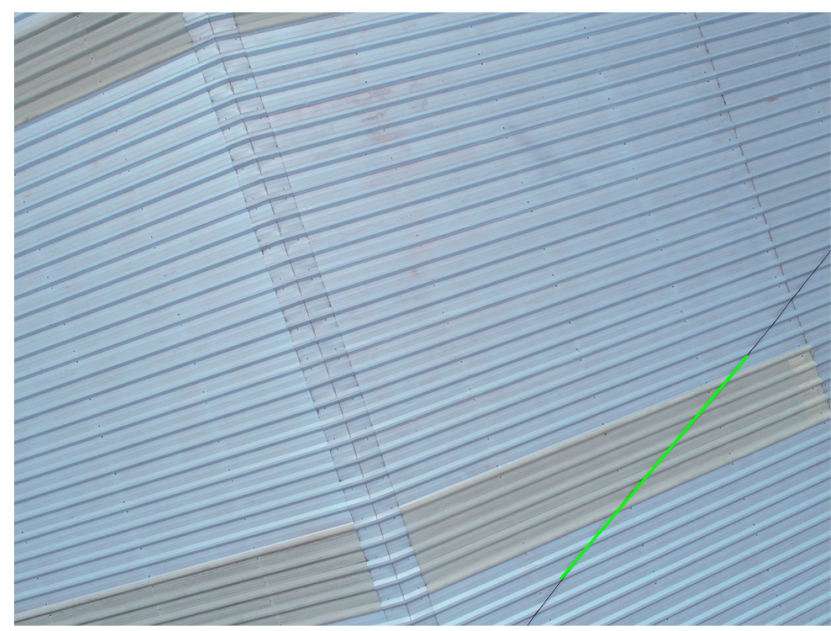

Fig. 4. Example of the false positive result.

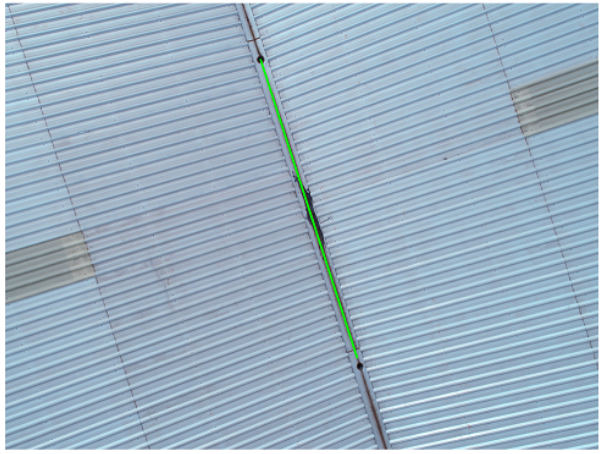

(a)

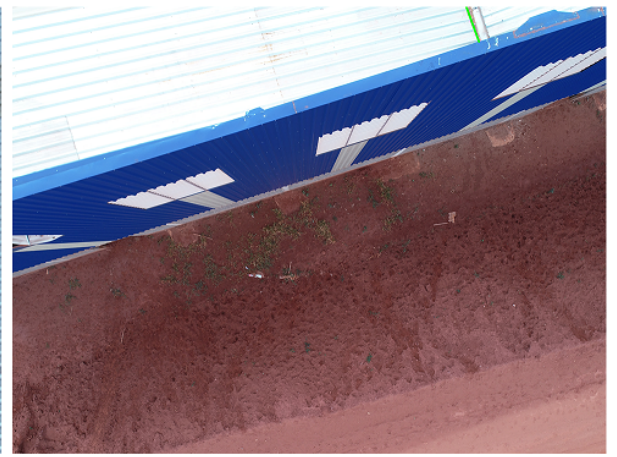

(b)

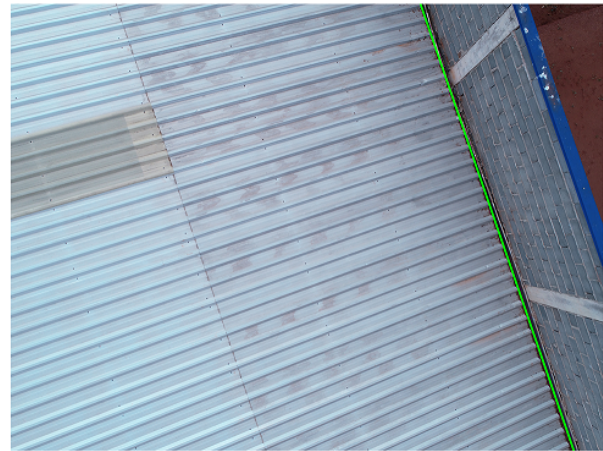

(c)

Fig. 5. Examples of the rain gutters detected.

Coordenação de Aperfeiçoamento de Pessoal de Nível Superior - Brazil (CAPES) - Finance Code 001.

\section{REFERENCES}

[1] A. L. Cavalcante, S. M. F. Brito, and A. S. Benzaken, "Monitoramento dos casos de dengue, febre de chikungunya e febre pelo vírus zika até a semana epidemiológica," 2018, [accessed Feb 13]. [Online]. Available: http://portalarquivos2.saude.gov.br/images/pdf/2018/ janeiro/23/Boletim-2018-001-Dengue.pdf

[2] E. Duarte, G. D. S. Ferreira, and M. B. D. Turcato, "Monitoramento dos casos de dengue, febre de chikungunya e febre pelo vírus zika até a semana epidemiológica 7," 2018, [accessed Feb 13]. [Online]. Available: http://portalarquivos2.saude.gov.br/images/pdf/2018/marco/06/ 2018-008-Publicacao.pdf

[3] A. A. P. Neto, A. S. Souza, and J. H. Arruda, "Boletim de vigilância em saúde. boletim epidemiológico," 2018, [accessed Feb 13]. [Online]. Available: http://www.uberlandia.mg.gov.br/uploads/cms_ b_arquivos/18763.pdf

[4] E. M. G. Paula, R. A. Oliveira, and G. A. Correa, "Boletim de vigilância em saúde," 2018, [accessed Feb 13]. [Online]. Available: http://www.uberlandia.mg.gov.br/uploads/cms_b_arquivos/18854.pdf

[5] V. S. Santos, "Ciclo de vida do aedes aegypti," 2018, [accessed Feb 13]. [Online]. Available: https://brasilescola.uol.com.br/animais/ ciclo-vida-aedes-aegypti.htm

[6] A. Mendonça, "Brasil: 2 milhões de casos de doenças causadas pelo aedes aegypti," 2018, [accessed Feb 13]. [Online]. Available: https://www.tarobanews.com/noticias/ciencia-e-saude/ brasil-2-milhoes-de-casos-de-doencas-causadas-pelo-aedes-aegypti-pMW html
[7] Brasil, "Nota a imprensa: Papel dos agentes comunitários de saúde," 2018, [accessed Feb 13]. [Online]. Available: http://combateaedes.saude.gov.br/pt/profissional-e-gestor/ orientacoes/141-papel-dos-agentes-comunitarios-de-saude

[8] P. Anupa Elizabeth, S. M, P. Paulraj, S. Pandian, and B. Tyagi, "Identification and eradication of mosquito breeding sites using wireless networking and electromechanical technologies," 122014.

[9] M. Mehra, A. Bagri, X. Jiang, and J. Ortiz, "Image analysis for identifying mosquito breeding grounds," in 2016 IEEE International Conference on Sensing, Communication and Networking (SECON Workshops), June 2016, pp. 1-6.

[10] J. Livingston and R. Steele, "A crowdsensing algorithm for imputing zika outbreak location data," in 2017 IEEE 8th Annual Ubiquitous Computing, Electronics and Mobile Communication Conference (UEMCON), Oct 2017, pp. 334-340.

[11] A. Amarasinghe, C. Suduwella, L. Niroshan, C. Elvitigala, K. De Zoysa, and C. Keppetiyagama, "Suppressing dengue via a drone system," in 2017 Seventeenth International Conference on Advances in ICT for Emerging Regions (ICTer), Sep. 2017, pp. 1-7.

[12] T. Dias, V. Alves, H. Alves, L. Pinheiro, R. Pontes, G. Araujo, A. Lima, and T. Prego, "Autonomous detection of mosquito-breeding habitats using an unmanned aerial vehicle," in 2018 Latin American Robotic Symposium, 2018 Brazilian Symposium on Robotics (SBR) and 2018 Workshop on Robotics in Education, Nov 2018, pp. 351-356.

[13] J. Serra, Image Analysis and Mathematical Morphology. Book, 1982.

[14] R. C. Gonzalez and R. C. Woods, Processamento Digital de Imagens, 3rd ed. Pearson, 2010.

[15] R. Duda and P. E. Hart, "Use of the hough transformation to detect lines and curves in pictures," CACM, vol. 15, pp. 11-15, 1972. 